\title{
Decalcification of Breast Cancer Bone Metastases With EDTA Does Not Affect ER, PR, and HER2 Results
}

\author{
Suzanne C. van ES, MD, * Bert van der Vegt, MD, PhD, † Frederike Bensch, MD, PhD, * \\ Sophie Gerritse, MD, $\neq$ Erik J. van Helden, MD, $\neq$ Eline Boon, MD, $\S$ Lindsay Angus, MD, \| \\ Jelle Overbosch, MD, $\uparrow$ Catharina W. Menke-van der Houven van Oordt, MD, PhD, $\neq$ \\ Henk M. Verheul, MD, PhD, $\neq$ Carla M.L. van Herpen, MD, PhD, \& Agnes Jager, MD, PhD,\# \\ Sjoukje F. Oosting, MD, PhD, ${ }^{*}$ Elisabeth G.E. de Vries, MD, PhD,* \\ and Carolina P. Schröder, MD, PhD*
}

\begin{abstract}
In metastatic breast cancer (MBC), expression of estrogen receptor (ER), progesterone receptor (PR), and human epidermal growth factor receptor-2 (HER2) guides treatment selection. In case of bone-only metastatic disease, ER, PR, and HER2 status assessment may be hampered by decalcification. We aimed to determine the optimal decalcification method, and to study discordance of receptor expression between paired primary breast tumors and optimally decalcified bone metastases. First, decalcification was simulated using acetic acid, hydrochloric/formic acid, and EDTA on 12 primary breast carcinomas. ER, PR, and HER2 immunohistochemistry (IHC) and HER2 in situ hybridization (ISH) were assessed, before and after the 3 decalcification methods. EDTA was considered the optimal method, as it did not affect IHC and as ISH failed in only 1/16 cases. Hydrochloric/formic acid altered ER and PR results, and, with acetic acid and hydrochloric/formic acid, ISH failed in, respectively, $94 \%$ and $100 \%$. Second, ER, PR, and HER 2 IHC was performed in paired primary tumors and EDTA-decalcified bone metastases obtained from patients with first presentation of MBC. Clinically relevant discordance was defined as changed receptor status with treatment implications. Paired samples of 77 patients, participating in the IMPACT-MBC trial, were evaluable. Hormonal receptor expression change was clinically relevant in 6 patients $(7.9 \%)$ and HER2 expression change in 1
\end{abstract}

From the Departments of * Medical Oncology; †Pathology; $\uparrow$ Radiology, University Medical Center Groningen, University of Groningen, Groningen; \$Department of Medical Oncology, Amsterdam University Medical Center (UMC), VU Medical Center (VUmc), Cancer Center Amsterdam, Amsterdam; §Department of Medical Oncology, Radboud University Medical Center, Nijmegen; ||Departments of Medical Oncology and Radiology \& Nuclear Medicine, Erasmus MC Cancer Institute; and \#Department of Medical Oncology, Erasmus University Medical Center, Rotterdam, The Netherlands.

Conflicts of Interest and Source of Funding: Supported by the Dutch Cancer Society, grant 2012-5565. The authors have disclosed that they have no significant relationships with, or financial interest in, any commercial companies pertaining to this article.

Correspondence: Carolina P. Schröder, MD, PhD, Department of Medical Oncology, University Medical Center Groningen, University of Groningen, P.O. Box 30 001, Groningen 9700 RB, The Netherlands (e-mail: c.p.schroder@umcg.nl).

Copyright (c) 2019 Wolters Kluwer Health, Inc. All rights reserved. patient $(1.3 \%)$. This study shows that EDTA decalcification minimally affects receptor expression results. The incidence of clinically relevant discordance between the primary tumor and bone metastases is low. These findings support that bone biopsies can reliably be used to assess receptor status.

Key Words: breast cancer, bone metastases, decalcification, EDTA, immunohistochemistry, in situ hybridization

(Am J Surg Pathol 2019;00:000-000)

In metastatic breast cancer (MBC), discordance of characteristics such as expression of estrogen receptor (ER), progesterone receptor (PR), and human epidermal growth factor receptor-2 (HER2) between the primary tumor and metastasis can have therapeutic implications. ${ }^{1}$ The National Comprehensive Cancer Network guidelines for breast cancer state that first recurrence of disease should be biopsied to determine ER, PR, and HER2 status of the metastasis. ${ }^{2}$ False-negative ER and/or PR results occur, but there may also be true receptor discordance between the primary tumor and metastases due to cause of loss of expression, that is, conversion. ${ }^{2,3} \mathrm{Ap}-$ proximately $70 \%$ of all advanced breast cancer patients develop bone metastases during the course of their disease, ${ }^{4}$ and $51 \%$ of patients with a history of breast cancer suffer a first relapse with bone-only disease. ${ }^{5} \mathrm{~Pa}$ tients' compliance with bone biopsy is acceptable, and the prevalence of complications is low. Antigenicity of the tumor cells, however, may be affected by decalcification methods. ${ }^{6,7}$ Decalcification is needed to enable sectioning of the specimen. Conflicting results are reported with regard to the reliability of pathologic assessment in decalcified tissue. A study that used immunohistochemistry (IHC) for HER2 and HER2 fluorescent in situ hybridization (FISH) in 149 breast cancer bone metastases showed an excellent success rate, with interpretable FISH analysis in $85 \%$ of cases and high concordance between IHC and FISH. ${ }^{7}$ In 107 patients with MBC, rather high discordance rates in ER, PR, and HER2 results between the primary tumor and bone metastasis were found 
$(20.5 \%, 43.9 \%$, and $6.9 \%$, respectively). This was in line with the results of previous studies in nonosseous metastases. ${ }^{1}$ However, several in vitro experiments showed a negative effect of decalcification on the immunoreactivity of breast cancer biomarkers and HER2 FISH. ${ }^{8-11}$ One explanation for the contradictive results could be the various decalcification methods that are used, with different decalcification agents and decalcification time spans. Currently, there is no gold standard for decalcification. As false-negative results may clearly affect treatment decision and patient outcome in $\mathrm{MBC}$, it is of great importance to clarify the reliability of IHC of bone metastases after decalcification.

The aims of this exploratory study were to, first, determine the optimal decalcification method by investigating which decalcification method has the least influence on ER, PR, and HER2 expression and, second, to study the discordance rates of ER, PR, and HER2 expression between paired primary breast tumors and bone metastases decalcified using the optimal method.

\section{MATERIALS AND METHODS}

\section{Aim 1. Assess the Optimal Decalcification Method}

\section{Tissue Selection}

Redundant material of 20 different primary breast cancers was collected, regardless of IHC characteristics. According to Dutch law and institutional guidelines, no informed consent was needed. The objection register of the institutions were consulted; none of the patients had objected to the use of redundant material for scientific purposes.

\section{Tumor Histology}

All tumors were fixed in buffered formalin for 24 to 48 hours, and, of each tumor, 7 blocks were obtained. One was processed according to standard. The other blocks were subjected to EDTA (Titriplex III 20\%, phosphate-buffered formalin $4 \%$ and sodium hydroxide $2 \%$ in distilled water [pH 7.0 to 7.2]), hydrochloric/formic acid (sodium citrate $0.8 \%$, formic acid $2 \%$, hydrochloric acid $1.5 \%$ in distilled water), or acetic acid (3.5-4\% formaldehyde and 10\% acetic acid in distilled water) for 24 or 48 hours.

All samples were embedded in paraffin. From each block, 3 cores were randomly included in a tissue microarray. Three-micrometer sections were cut, and ER (SP1; Ventana, Illkirch, France), PR (1E2; Ventana), and HER2 (SP3; Ventana) were stained with a Benchmark Ultra Automated stainer (Ventana) according to the manufacturer's manual. Antibodies were prediluted by the supplier. FISH (PathVysion HER2 DNA Probe kit, Abbott Molecular, IL) and silver in situ hybridization (ISH) (INFORM HER2 Dual ISH DNA Probe Cocktail; Ventana) assays were performed following the manufacturer's recommendations. All samples were scored by an experienced breast pathologist who was blinded to the decalcification method and time. ER, PR, and HER2 were scored according to the ASCO/CAP guidelines. ${ }^{12,13}$

\section{Statistical Analysis}

Descriptive statistics were used to evaluate the results of this decalcification experiment. The overall discordance rate (ODR) and the $95 \%$ confidence interval (CI), using the Wilson score method, were calculated.

\section{Aim 2. Assess the Discordance Rate between Primary Tumor and Bone Metastasis With the Optimal Decalcification Method}

\section{Tissue Selection}

The tumor tissues of patients enroled in the IMPACT-MBC trial (NCT01957332) between September 2013 and May 2018, who had undergone a bone metastasis biopsy $(\mathrm{n}=96)$, were selected. Of these, only cases with bone metastases that were decalcified using the optimal decalcification method, as assessed in the abovementioned experiment, and of which revised and paired data were available, were included $(n=77)$.

The IMPACT-MBC trial includes newly diagnosed MBC patients, with nonrapidly progressive disease, who were eligible for first-line systemic treatment. This multicenter study, conducted at the University Medical Center Groningen (UMCG), Amsterdam University Medical Center (UMC), location VU Medical Center (VUmc), Radboud University Medical Center (Radboud UMC), and Erasmus Medical Center (Erasmus MC), was approved by the Medical Ethical Committee of the University Medical Center Groningen and the Central Committee on Research Involving Human Subjects. All patients gave written informed consent for central evaluation of the primary tumor, biopsy of a metastatic lesion, and (central) assessment of the molecular characteristics of the metastasis. Here, we present an analysis of the standard pathologic assessments of the tumor tissue.

\section{Tumor Histology}

In all centers, bone biopsies were formalin fixed. In the UMCG and Radboud UMC, the specimens $(n=75)$ were decalcified using EDTA for 24 hours at room temperature, or, if the decalcification process was incomplete, for 48 hours. In the Erasmus MC, EDTA decalcification was applied, for $\sim 7$ hours, at $37^{\circ} \mathrm{C}(\mathrm{n}=10)$. In the Amsterdam-UMC location, VUMC sakura TDE30 (without electrolysis) was used to decalcify the tissue for a duration of at least 60 minutes and up to a maximum of 90 minutes; therefore, the tissues of these patients $(n=11)$ were excluded. IHC for ER, PR, and HER2, HER2 FISH, and HER2 SISH was performed according to the protocol described above. Both primary breast tumors and bone biopsies were centrally evaluated by a dedicated breast pathologist at the UMCG.

\section{Statistical Analysis}

The ODR and the 95\% CI, using the Wilson score interval, were calculated. McNemar test was used to compare the prevalence of ER, PR, and HER2 receptor status between the primary tumor and bone metastases. The percentage ER and PR expression of paired samples 
of the primary tumor and the bone metastasis were evaluated using the Wilcoxon Signed Rank test. $P$-value $<0.05$ was considered a statistically significant discordance. In case of multiple primary breast tumors, the most recent tumor was used. Clinically relevant discordance was defined as a change of receptor status, with treatment implications.

Data were analyzed using IBM SPSS statistics version 23 (SPSS Inc., Chicago, IL).

\section{RESULTS}

\section{Aim 1. Optimal Decalcification Method}

Of the 20 fresh residual primary breast cancer tissues, 8 did not contain any invasive tumor. After processing, 1 of 12 cases was excluded for IHC analyses, because the standard processed (control) sample lacked tumor cells. One other sample was excluded for ER and PR analyses because of the same reason; this case was available for HER2 analysis. The 10 remaining tumors for all IHC analyses were ER+PR+HER2 $-(n=6), E R+P R$ -HER2- $(\mathrm{n}=1), \mathrm{ER}-\mathrm{PR}-\mathrm{HER} 2+(\mathrm{n}=1)$, and ER-PR -HER2- $(\mathrm{n}=2)$. The extra tumor for the HER2 IHC was HER2-. For SISH analyses, 1/12 tissues did not contain tumor cells in the control sample, and, in 3/12, SISH failed on the control sample, leaving 8 tissues for ISH analysis. For FISH analyses, in 6/12 tissues, FISH failed on the control sample. For both IHC and ISH analyses, both blocks, decalcified for 24 and 48 hours, were studied.

In Table 1, the effects of the 3 decalcification agents on ER, PR, and HER2 IHC are summarized. No IHC receptor status result was altered by acetic acid; however, technical SISH failure occurred in 15/16 cases with interpretable control ISH, and FISH failed in 12/12 cases. The use of hydrochloric/formic acid resulted in false-negative ER staining in 3 of 7 ER-positive tumors (ODR 30\%; 95\% CI, 11\%-60\%), which showed 60\%, 60\%, and $70 \%$ ER staining in the control block. False-negative PR staining occurred in 2/6 PR-positive tumors (ODR $20 \%$; $95 \%$ CI, $6 \%-51 \%$ ), which showed $20 \%$ and $40 \%$ PR positivity in the control. Decalcification for 48 hours with hydrochloric/formic acid decreased HER2 IHC from 3+ into 2+ (1/1). Technical SISH and FISH failure was observed in all cases. EDTA altered no IHC receptor

TABLE 1. Comparison of the Effects of Acetic Acid, Hydrochloric/Formic Acid, and EDTA on IHC and the Clinical Relevance of the Effects, Showing that EDTA is the Best Decalcification Method

\begin{tabular}{lccc}
\hline & $\begin{array}{c}\text { Acetic Acid } \\
\text { Median, Mean } \\
\text { (Range) }\end{array}$ & $\begin{array}{c}\text { Hydrochloric/Formic } \\
\text { Acid Median, Mean } \\
\text { (Range) }\end{array}$ & $\begin{array}{c}\text { EDTA } \\
\text { Median, Mean } \\
\text { (Range) }\end{array}$ \\
\hline ER & $0 \%, 2.5 \%$ & $-5 \%,-21 \%$ & $0 \%,-0.5 \%$ \\
PR & $(-30 \%$ to $40 \%)$ & $(-70 \%$ to $0 \%)$ & $(-40 \%$ to $40 \%)$ \\
HER2 & $0 \%, 0.5 \%$ & $-5 \%,-14.5 \%$ & $0 \%,-1.5 \%$ \\
& $(-60 \%$ to $60 \%)$ & $(-40 \%$ to $5 \%)$ & $(-40 \%$ to $60 \%)$ \\
\end{tabular}

status result, SISH failed in 1 of 16 cases, and FISH showed $100 \%$ concordance with the control samples.

EDTA was, therefore, considered the most optimal decalcification method.

\section{Aim 2. Discordance Rate Between Primary Tumor and Bone Metastasis With Optimal Decalcification Method}

\section{Tumor Characteristics}

Figure 1 shows the CONSORT flow diagram, describing the selection process of the tumor tissue from the IMPACT-MBC study. All 77 metastatic tissues were obtained from female patients, with a median tumor relapse time of 75.8 months (range, 0.6 to $293.5 \mathrm{mo}$ ). For the evaluation of percentage of ER and PR staining in paired primary tumors and bone metastases, 1 extra case was excluded, because no exact percentages could be given for ER and PR staining of the metastasis. The histologic subtype of the primary tumor was invasive carcinoma of no special type in $72.7 \%$, lobular carcinoma in $26 \%$, and carcinoma with apocrine differentiation in $1.3 \%$ of cases.

\section{ER Staining}

Table 2 shows the ER status of the primary tumors and metastases. Five of 77 patients had an ER-positive primary tumor and an ER-negative bone metastasis, and 1

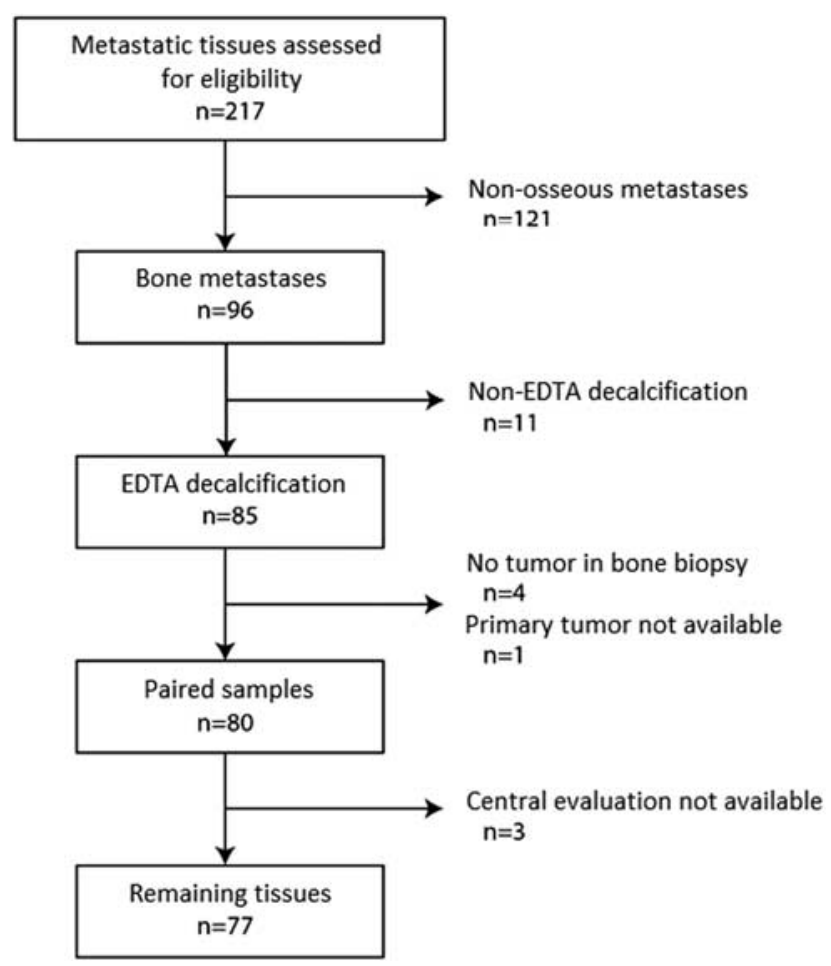

FIGURE 1. CONSORT diagram reflecting the selection process of the tumor tissue from the IMPACT breast study. Of the 217 included patients, between September 2013 and May 2018, 96 patients underwent bone biopsy, of which 77 were evaluable for this analysis. 
TABLE 2. Concordance and Discordance of ER Status of Primary Breast Tumors and Bone Metastases

\begin{tabular}{lccc}
\hline & \multicolumn{3}{c}{$\mathbf{n}(\%)$} \\
\cline { 2 - 4 } & $\begin{array}{c}\text { Bone Metastasis } \\
\text { ER- }\end{array}$ & $\begin{array}{c}\text { Bone Metastasis } \\
\text { ER+ }\end{array}$ & Total \\
\hline Primary ER- & $9(11.7)$ & $1(1.3)$ & $10(13)$ \\
Primary ER+ & $5(6.5)$ & $62(80.5)$ & $67(87)$ \\
Total & $14(18.2)$ & $63(81.8)$ & $77(100)$ \\
\hline
\end{tabular}

patient had an ER-negative primary tumor and ERpositive bone metastasis; ODR $7.8 \%$ (95\% CI, 4\%-16\%) $(P=0.219)$.

The median ER expression was $100 \%$ for both the primary tumors and the bone metastases; the mean ER expression was $81 \%$ for the primary tumors and $73 \%$ for the bone metastases. ER expression of paired primary tumor and bone metastasis was discordant in 31 of the 76 patients $(P=0.044)$, with a median change in IHC expression of $-20 \%$ (range, $-100 \%$ to $+99 \%$ ). An increase in ER expression was seen in $17 \%$ of patients (median $20 \%$, range $5 \%$ to $99 \%$ ). A decline in ER expression was observed in $24 \%$ of patients (median $25 \%$, range $20 \%$ to $100 \%$ ). In $59 \%$ of cases, the percentage of ER expression remained the same (Fig. 2).

\section{PR Staining}

Table 3 shows the PR status of the primary tumors and metastases; $15.6 \%$ (12/77) of patients had PR-positive primary tumor and negative bone metastasis biopsy, and $5.2 \%(4 / 77)$ of patients had PR-negative primary tumor, with positive PR staining of the bone metastasis biopsy; ODR $20.8 \%(95 \% \mathrm{CI}, 13 \%-31 \%)(P=0.077)$.

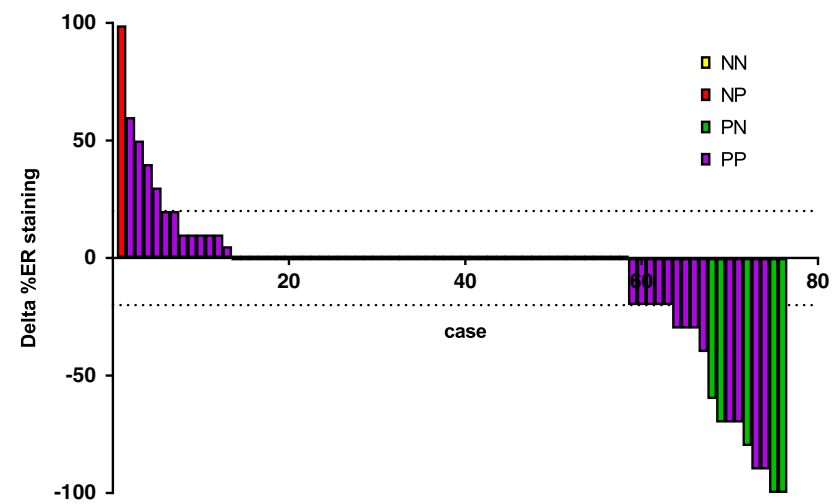

FIGURE 2. Waterfall plot of the change in ER expression between the primary tumor and metastasis in 76 paired cases (1 was excluded because no exact ER percentages could be given). The horizontal dotted lines are drawn at $-20 \%$ and $20 \%$. The cases with receptor conversion showed an expression difference of at least $60 \%$. NN indicates ER-negative primary tumor and ER-negative metastasis; NP, ER-negative primary tumor and ER-positive metastasis; PN, ER-positive primary tumor and ER-negative metastasis; PP, ER-positive primary tumor and ER-positive metastasis.
TABLE 3. Concordance and Discordance of PR Status of Primary Breast Tumors and Bone Metastases

\begin{tabular}{lccc}
\hline & \multicolumn{3}{c}{$\mathbf{n}(\%)$} \\
\cline { 2 - 4 } & $\begin{array}{c}\text { Bone Metastasis } \\
\text { PR- }\end{array}$ & $\begin{array}{c}\text { Bone Metastasis } \\
\text { PR+ }\end{array}$ & Total \\
\hline Primary PR- & $13(16.9)$ & $4(5.2)$ & $17(22.1)$ \\
Primary PR+ & $12(15.6)$ & $48(62.3)$ & $60(77.9)$ \\
Total & $25(32.5)$ & $52(67.5)$ & $77(100)$ \\
\hline
\end{tabular}

The median PR expression was $70 \%$ for the primary tumors and $40 \%$ for the bone metastases; the mean expression was, respectively, $57 \%$ and $44 \%$. Paired testing of the percentage PR expression of the primary tumor and the bone metastasis $(\mathrm{n}=76)$ was discordant in 50 of the 76 patients $(P=0.009)$, with a median change of IHC expression of $-12.5 \%$ (range $-100 \%$ to $+100 \%$ ). An increase in PR expression was seen in $22 \%$ of cases (median $22.5 \%$, range $2 \%$ to $100 \%$ ), a decline in $43 \%$ of cases (median $40 \%$, range $5 \%$ to $100 \%$ ), and, in $34 \%$ of cases, the percentage of PR staining remained the same (Fig. 3).

\section{HER2 Staining}

Table 4 shows the HER 2 status of the primary tumors and metastases. One of the 77 patients had an HER2-positive primary tumor and HER2-negative bone metastasis $(P=0.500 ;$ ODR $1.3 \%, 95 \% \mathrm{CI}, 1 \%-7 \%)$. In this case, the primary tumor had an IHC score of $3+$ (therefore, no ISH was performed), and the bone metastasis was IHC $2+$ with no ISH amplification.

Paired testing of the IHC score of the primary tumors and the metastasis $(n=77)$ showed no difference. In $25 \%$ of cases, the metastasis had a higher IHC score than the primary

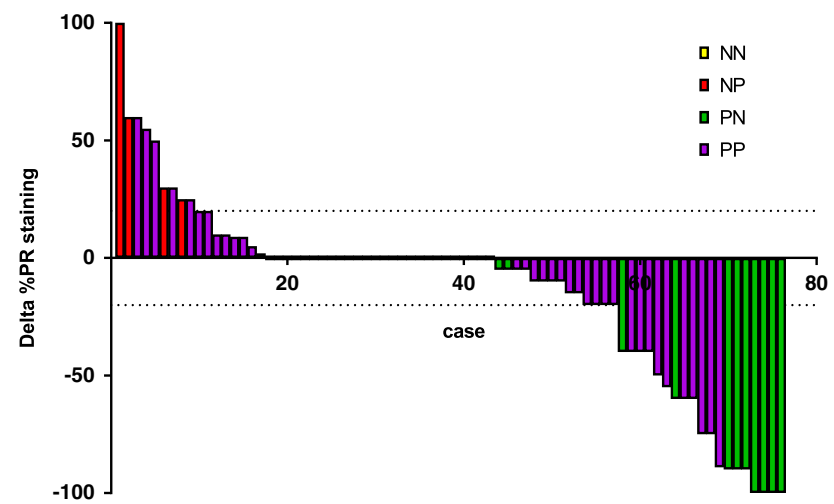

FIGURE 3. Waterfall plot of the change in PR staining between the primary tumor and metastasis in 76 paired cases (1 case was excluded because no exact PR percentages could be given). The horizontal dotted lines are drawn at $-20 \%$ and $20 \%$. The cases with receptor conversion showed an expression difference of $2 \%$ to $100 \%$. NN indicates PR-negative primary tumor and PR-negative metastasis; NP, PR-negative primary tumor and PR-positive metastasis; PN, PR-positive primary tumor and PRnegative metastasis; PP, PR-positive primary tumor and PR-positive metastasis. 
TABLE 4. Concordance and Discordance of HER2 Status of Primary Breast Tumors and Bone Metastases

\begin{tabular}{lccc}
\hline & \multicolumn{3}{c}{ n (\%) } \\
\cline { 2 - 4 } & $\begin{array}{c}\text { Bone Metastasis } \\
\text { HER2- }\end{array}$ & $\begin{array}{c}\text { Bone Metastasis } \\
\text { HER2+ }\end{array}$ & Total \\
\hline $\begin{array}{c}\text { Primary } \\
\text { HER2- }\end{array}$ & $66(85.7)$ & 0 & $66(85.7)$ \\
Primary & $2(2.6)$ & $9(11.7)$ & $11(14.3)$ \\
$\quad$ HER2+ & $68(88.3)$ & $9(11.7)$ & $77(100)$ \\
Total & & & \\
\hline
\end{tabular}

tumor; in 18\%, the score was lower; and, in 57\%, the score remained the same. ISH gave equivocal results (Fig. 4).

\section{Clinical Relevance}

In six of the 77 patients $(7.9 \%)$, clinically relevant discordance of both ER and PR was observed; in five cases, hormonal receptor (HR) status changed from positive to negative; and, in one, from negative to positive. One of 5 patients, in whom HR status converted from positive (ER $100 \%$, PR $90 \%$ ) on the primary tumor to HR negative on the bone metastasis, also underwent a nonosseous biopsy 3 weeks after the bone biopsy; this was ER negative and PR positive $(60 \%)$. One of 77 patients $(1.3 \%)$ had HER2positive primary breast tumor, while the bone metastasis was HER2 negative. This patient is one of the 5 cases in which HR was discordant, with the primary tumor being HR negative and the metastasis being HR positive. Altogether, in $7.9 \%$ of MBC patients, the discordance affected treatment choices. The disease-free survival (time between the primary tumor and the metastasis) was not related to the presence or absence of clinically relevant discordance, with $P=0.82$ for the entire group, versus patients with a change of ER status and $P=0.93$ for the entire group, versus patients' with a change of PR status (Fig. 5).

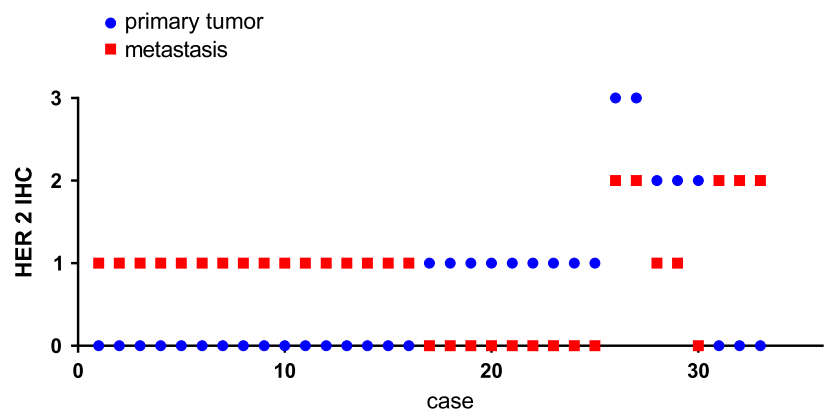

FIGURE 4. Visualization of the cases in which a change of human epidermal growth factor-2 immunohistochemistry (HER2 IHC) score was observed (33/77). The 33 cases are depicted on the $x$-axis and the HER2 score can be appreciated from the $y$-axis. For every case, the HER2 score for both the primary tumor (blue dot) and metastasis (red box) is visualized. In 25 cases, the score was 0 on the primary tumor and 1 on the metastasis or vice versa. In the 8 remaining cases, the score was $3+$ on the primary tumor and $2+$ on the bone metastases $(n=2)$; a decline was seen from $2+$ to either $1+(n=2)$ or to 0 $(n=1)$, and, in 3 cases, HER2 score went up from 0 to $2+$.

\section{DISCUSSION}

This exploratory analysis shows that decalcification with EDTA has very little effect on ER, PR, and HER2 results of breast cancer metastases in bone, measured by IHC or ISH. After EDTA decalcification of bone metastases, the clinically relevant discordance rate of these receptors at first presentation of $\mathrm{MBC}$ is low.

To our knowledge, this is the first study investigating the most optimal decalcification method and its impact on IHC and ISH results, by translating a methodological experiment to a large prospective data set. These findings support the regular use of bone biopsies in clinical practice to assess receptor status, if the most optimal decalcification method is used. This is clearly of relevance in bone-only disease, which is common in MBC.

ASCO/CAP recognizes that preanalytic variables must be controlled to ensure that assay results reflect tumor ER, PR, and HER2 status. In this setting, much attention is focused in the ASCO/CAP guideline on optimal fixation methods. Although the guideline states that samples with decalcification artifacts should be rejected, and that samples that were decalcified using a strong acid may be rejected and testing should be repeated on a separate sample, no specific recommendations for optimal bone decalcification have been made. ${ }^{12,13}$

With the optimal decalcification method with EDTA, we showed, in our newly diagnosed MBC patients, that discordance between primary breast cancer and bone metastases was found for ER, PR, and HER2 status in $7.9 \%, 20.8 \%$, and $1.3 \%$ of cases, respectively. The ER, PR, and HER2 discordance rate in our population is less than previously reported in other studies, namely $20.5 \%, 43.9 \%$, and $6.9 \%{ }^{4}$ and $29.3 \%, 42.7 \%$, and $8.5 \%$, respectively. ${ }^{14}$ In both studies, EDTA was used as the decalcification method. Moreover, in recent studies with nonosseous metastases, higher discordance rates were reported. ${ }^{14-16}$ Our lower discordance rate may be explained by the fact that our study included only patients at first presentation of metastatic disease. It is clear by now that breast cancer characteristics may change also during the course of the metastatic disease and following noncurative treatment. Clonal expansion, particularly in case of a heterogenous primary tumor, might explain discordance. ${ }^{17}$ Furthermore, we only included patients without rapid progression, selecting the more indolent tumor type. In addition, true conversion might be in play, or possibly a second primary tumor with distinct characteristics as the source of the metastases. In any case, and despite the low frequency of receptor discordance observed in our study, it can have clear clinical implications. This underlines the necessity to evaluate receptor status during the course of the disease.

In our prospective cohort, only $14.3 \%$ of the women had an HER2-positive primary tumor. Previously, HR and HER2 of bone metastases were compared with the primary breast cancer in a retrospective cohort. In that study, merely $7.3 \%$ of 107 patients had an HER2-positive primary breast cancer. ${ }^{18}$ As these data concern relatively small subsets of patients, it would be of interest if results could be confirmed in a larger cohort with HER2-positive 


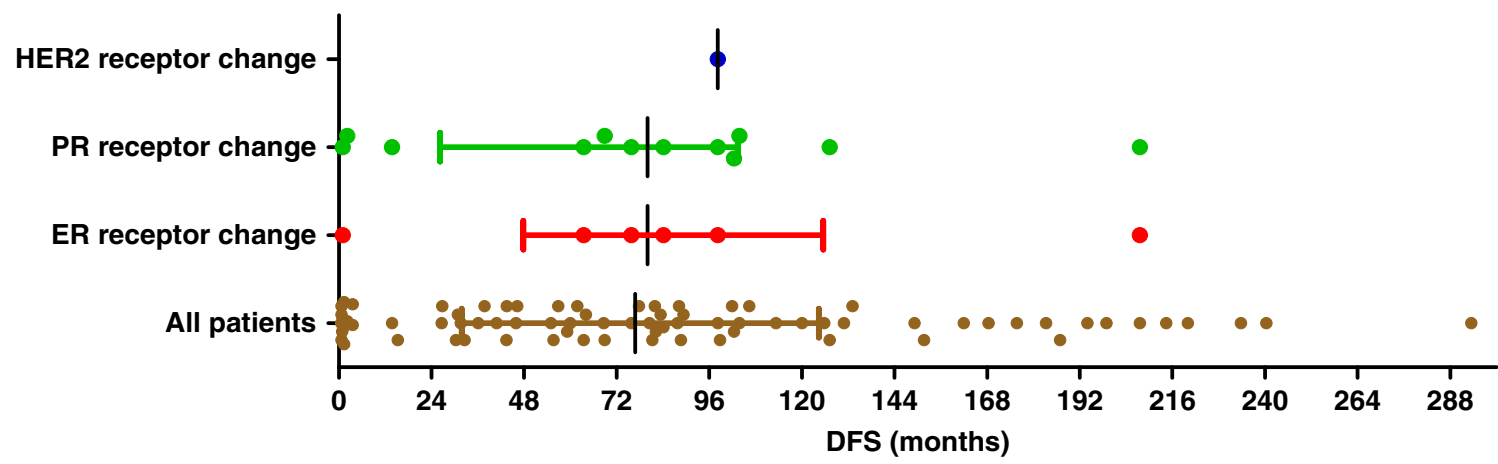

FIGURE 5. Dot plot of the time between the diagnosis of the primary breast tumor and the bone biopsy of the metastasis (relapse time) in months. The brown dots represent all patients in this study with median and interquartile range. Subgroups of patients with change of ER status (patients in red dots), change of PR status (green dots), and change of HER2 status (blue dot); the median relapse time of patients with ER or PR change is equal to the entire group with overlapping interquartile ranges.

disease. In only 1 patient with clinical relevant discordance of receptor status between the primary breast tumor and bone metastasis, another nonosseous biopsy of a metastasis was available. This additional biopsy confirmed the loss of ER; however, contrary to the bone metastasis, it did express PR, which can be explained by either heterogeneity or false-negative results of the bone biopsy. For the other 6 patients with clinically relevant conversion, no other biopsy was available to either confirm or reject these findings.

Although the results of our study show that HER2 status can reliably be determined after decalcification, separating biopsies into softer and harder fragments to avoid possible artifact by decalcification of tumor material may also be considered.

In conclusion, in this exploratory analysis, we showed that decalcification with EDTA does not interfere with ER, PR, and HER2 expression in breast cancer bone metastases. The incidence of clinically relevant discordance is low at first presentation of MBC. Our findings support that bone metastases biopsies can reliably be used to assess receptor status in clinical practice.

\section{ACKNOWLEDGMENTS}

The authors acknowledge the technicians E. Bleuel and J. van Dekken for their technical support and the involved radiologists for their proficient bone biopsies.

\section{REFERENCES}

1. Aurilio G, Monfardini L, Rizzo S, et al. Discordant hormone receptor and human epidermal growth factor receptor 2 status in bone metastases compared to primary breast cancer. Acta Oncol. 2013;52:1649-1656.

2. National Comprehensive Cancer Network (NCCN) guideline version 1.2019 breast cancer. 2019. Available at: https://www.nccn.org/ professionals/physician_gls/pdf/breast.pdf. Accessed June 26, 2019.

3. Curtit E, Nerich V, Mansi L, et al. Discordance in estrogen receptor status, progesterone receptor status, and HER2 status between primary breast cancer and metastasis. Oncologist. 2013;18:667-674.

4. Galasko C. The anatomy and pathways of skeletal metastases. In: Weiss L, Gilbert A, eds. Bone Metastases. Boston, MA: GK Hall; 1981:49-63.
5. Coleman RE, Rubens RD. The clinical course of bone metastases from breast cancer. Br J Cancer. 1987;15:61-66.

6. Simmons C, Miller N, Geddie W, et al. Does confirmatory tumor biopsy alter the management of breast cancer patients with distant metastases. Ann Oncol. 2009;20:1499-1504.

7. Zustin J, Boddin K, Tsourlakis MC, et al. HER-2/neu analysis in breast cancer bone metastases. J Clin Pathol. 2009;62:542-546.

8. Clark BZ, Yoest JM, Onisko A, et al. Effects of hydrochloric acid and formic acid decalcification on breast tumor biomarkers and HER2 fluorescence in situ hybridization. Appl Immunohistochem Mol Morphol. 2019;27:223-230.

9. Schrijver WA, van der Groep P, Hoefnagel LD, et al. Influence of decalcification procedures on immunohistochemistry and molecular pathology in breast cancer. Mod Pathol. 2016;29:1460-1470.

10. Maclary AD, Mohanty SK, Bose S, et al. Effect of hydrochloric acid decalcification on expression pattern of prognostic markers in invasive breast carcinomas. Appl Immunohistochem Mol Morphol. 2017;2:144-149.

11. Gertych A, Mohan S, Maclary S, et al. Effects of tissue decalcification on the quantification of breast cancer biomarkers by digital image analysis. Diagn Pathol. 2014;9:213-222.

12. Hammond MEH, Hayes DF, Dowsett M, et al. American Society of Clinical Oncology/College of American Pathologists guideline recommendations for immunohistochemical testing of estrogen and progesterone receptor in breast cancer. J Clin Oncol. 2010;28: 2784-2795.

13. Wolff AC, Hammond MEH, Allison KH, et al. Human epidermal growth factor receptor 2 testing in breast cancer: American Society of Clinical Oncology/College of American Pathologists Clinical Practice Guideline Focused Update. J Clin Oncol. 2018;36:2105-2122.

14. Schrijver WAME, Suijkerbuijk KPM, an Gils CH, et al. Receptor conversion in distant breast cancer metastases: a systematic review and meta-analyses. JNCI Natl Cancer Ins. 2018;6:568-580.

15. Matsumoto A, Takahashi M, Hayashida T, et al. Changing ER, PgR and HER2 status between primary and recurrent breast cancer. J Clin Oncol. 2012;30(suppl 27):92.

16. Aurilio G, Disalvatore D, Pruneri G, et al. A meta-analysis of oestrogen receptor, progesterone receptor and human epidermal growth factor receptor 2 discordance between primary breast cancer and metastases. Eur J Cancer. 2014;50:277-289.

17. Juric D, Castel P, Griffith M, et al. Convergent loss of PTEN leads to clinical resistance to a $\mathrm{PI}(3) \mathrm{K} \alpha$ inhibitor. Nature. 2015;518: 240-244.

18. Fujita T, Sawaki M, Hattori M, et al. Receptor discordance in breast cancer recurrence: Is re-biopsy a necessity? Thirty-Fifth Annual CTRC-AACR San Antonio Breast Cancer Symposium, December 4-8, 2012; Abstract P1-02-02. 